\title{
Comunidades de roedores nocturnos en un ecotono de matorrales micrófilos y zacatal gipsófilo en San Luis Potosí, México
}

\author{
Nocturnal rodents at the edge of a microphyllous shrubland and gypsophilous grassland in San \\ Luis Potosí, Mexico
}

\author{
Jaime Luévano ${ }^{1,2 *}$, Eric Mellink², Mónica E. Riojas-López y José Luis Flores-Flores ${ }^{4}$ \\ ${ }^{1}$ Instituto Potosino de Investigación Científica y Tecnológica, Ciencias Ambientales. Camino a la presa 2055, 78216 San Luis Potosí, San Luis Potosí, \\ México \\ ${ }^{2}$ Departamento de Biología de la Conservación. Centro de Investigación Científica y Educación Superior de Ensenada. Km. 107 Carretera Tijuana- \\ Ensenada, 22860 Ensenada, Baja.California, México \\ ${ }^{3}$ Departamento de Ecología. Centro Universitario de Ciencias Biológicas y Agropecuarias. Universidad de Guadalajara. Km. 15.5 Carretera \\ Guadalajara-Nogales, 45100 Zapopan, Jalisco, México \\ ${ }^{4}$ Instituto de Investigaciones en Zonas Desérticas, Universidad Autónoma de San Luis Potosí. Altair 200, Fracc. Del Llano, 78377 San Luis Potosí, San \\ Luis Potosi, México \\ *Correspondencia: jluevano@cicese.mx
}

\begin{abstract}
Resumen. En el presente estudio se compara la abundancia y riqueza entre los roedores nocturnos de un zacatal gipsófilo y los de 3 matorrales micrófilos, en el norte de San Luis Potosí. Los muestreos se llevaron a cabo en sitios con distintas características edáficas y de estructura de la vegetación, en la estación lluviosa y en la seca de los años 2003 y 2004. Como estimador del tamaño poblacional se utilizó el número de individuos capturados en cada periodo. Se capturaron 414 individuos de 12 especies de las familias Heteromyidae y Muridae. Se concluye que: 1) las comunidades de roedores en cada sitio fueron diferentes en función de la estructura vegetal y cambiaron de manera notable entre el zacatal y los 3 sitios de matorral adyacentes; 2) dichas comunidades reflejaron el mosaico de la vegetación más que la época del año; 3 ) los tres tipos de matorral mantuvieron su identidad individual a lo largo del año, y 4) la baja precipitación y/o el pastoreo, a través de la reducción de la cobertura vegetal sobre suelos gipsófilos afectó a los roedores.
\end{abstract}

Palabras clave: abundancia, riqueza de roedores, Heteromyidae, Muridae.

\begin{abstract}
In this study we compared abundance and species richness of nocturnal rodents at the edge of gypsophilous grassland on an exposed gypsum substrate and 3 sites of microphilous shrubland, in northern San Luis Potosí, Mexico. We sampled at sites that differed in edaphic characteristics and vegetation structure, in the wet and dry seasons of 2003 and 2004. As a population estimator we used the number of individuals captured in each sampling period. We captured 414 individuals of 12 species of Heteromyidae and Muridae. We concluded that 1) rodent communities were different among sites as a function of vegetation structure, and varied notably between grassland and adjacent shrublands: 2) such communities reflected the vegetation mosaic more than season of the year: 3) the three shrubland types maintained their identity throughout the year; y 4) low precipitation and/or grazing, through the reduction of plant cover, on gypsophilous soils afected the rodents.
\end{abstract}

Key words: abundance, species richness, Heteromyidae, Muridae.

\section{Introducción}

En ambientes áridos, los factores abióticos, entre otros los atributos físicos del suelo, determinan en gran medida la composición de comunidades vegetales (Meyer et al., 1992; Guo et al., 1995). Las características edáficas de las grandes extensiones de terrenos yesosos de la parte árida de los estados de San Luis Potosí, Coahuila y Nuevo León, condicionan la vegetación presente. Se forma un mosaico

Recibido: 03 julio 2006; aceptado: 05 septiembre 2007 de vegetación que se relaciona con el suelo y el relieve. Donde aflora el yeso, la comunidad vegetal es un pastizal dominado por los zacates gipsófilos, perennes y endémicos, Muhlenbergia purpusii y Bouteloua chasei (Rzedowski, 1955). Cuando el sustrato yesoso está cubierto por material aluvial calcáreo, aparecen matorrales micrófilos conformados por especies incapaces de colonizar y sobre vivir sobre el yeso expuesto (Meyer et al., 1992), lo que conduce a que en estos paisajes de suelos yesíferos se produzcan mosaicos de vegetación con ecotonos bastante claros. 
En general, las características del hábitat determinan las comunidades animales. La influencia del macrohábitat es particularmente clara en el condicionamiento de las comunidades de roedores (Rosenzweig y Winakur, 1969; Kay y Whitford, 1978; Price, 1978; Bock et al., 1984; Price y Waser, 1984; Brown y Heske, 1990; Jones y Longland, 1999; Mellink y Valenzuela, 1995; Jorgensen, 2004; Riojas-López, 2006). Las comunidades de animales dependen de la calidad del microhábitat, por lo que se esperaría encontrar variaciones en la densidad y riqueza de roedores en manchones de hábitat con diferentes características. Sin embargo, esto parece poco respaldado por la información existente (Jorgensen, 2004).

No obstante la importancia de los factores edáficos para condicionar la comunidad vegetal, los disturbios antropógenos (como el pastoreo) afectan la composición de esas comunidades. Cuando se modifican la estructura y composición de la vegetación también se afecta la abundancia y riqueza específica de la fauna (Mellink y Valenzuela, 1992, 1995). Un disturbio antropogénico muy común en zonas áridas y semiáridas, incluyendo las del centro-norte de México, es el sobrepastoreo por herbívoros domesticados que en ocasiones, junto con los herbívoros silvestres, pueden causar cambios drásticos en la estructura de la vegetación (Fleischner, 1994; Archer, 1995; Mellink, 1995a). Sin embargo, se ha indicado que la composición de los zacatales de suelos gipsófilos es estable aún bajo condiciones de sobrepastoreo (Meyer y García-Moya, 1989). En el sur del desierto chihuahuense, los zacatales gipsófilos se han mantenido libres de arbustos a pesar de la fuerte presión de pastoreo ejercido durante los últimos 50 años, posiblemente debido al estrés tan severo que el yeso impone sobre las comunidades vegetales (Meyer et al., 1992).

En general, cuando las comunidades vegetales cambian espacialmente de manera gradual, el recambio en las especies de roedores es de intergradación. En contraste, los bordes entre comunidades vegetales condicionadas edáficamente pueden ser abruptos, como en la transición entre el zacatal gipsófilo y el matorral. En estos casos se esperaría que la transición entre comunidades de roedores sea también abrupta.

En El Manantial, una explanada yesosa de unas 340 ha en el norte de San Luis Potosí, dentro del municipio de Vanegas, hay un zacatal abierto virtualmente desprovisto de arbustos, salvo en sitios muy localizados. Esta zona de zacatal gipsófilo contrasta con los alrededores, donde el suelo tiene una cubierta aluvial calcárea con grandes extensiones de matorral xerófilo intercalado con pequeños manchones de zacatal. La explanada, además, es el sitio en el que se encuentra la colonia sureña más grande de perrito llanero mexicano Cynomys mexicanus (Ceballos et

\section{al., 1993; Yeaton y Flores, 2006).}

Esta zonación de la vegetación, vinculada con las restricciones edáficas a la invasión de arbustos, permite tener un mosaico de vegetación adecuado para estudiar las interacciones entre la vegetación y la comunidad de roedores nocturnos respectiva. En concordancia, la hipótesis del presente estudio es que la composición y abundancia de las comunidades de roedores nocturnos cambia de forma abrupta entre el fragmento de pastizal y los matorrales adyacentes, los cuales son menos diferentes entre sí en la comunidad de roedores que habita en ellos.

\section{Materiales y métodos}

Durante 2003 y 2004 se llevó a cabo un estudio del zacatal gipsófilo y de diferentes matorrales micrófilos en El Manantial, Vanegas, San Luis Potosí, México (entre $24^{\circ} 06^{\prime} 20^{\prime \prime}$ y $24^{\circ} 08^{\prime} 47^{\prime \prime} \mathrm{N}$ y $100^{\circ} 54^{\prime} 35^{\prime \prime}$ y $100^{\circ} 56^{\prime} 00^{\prime \prime}$ $\mathrm{O})$, para determinar la composición y abundancia de las comunidades de roedores nocturnos. Los suelos del área son xerosoles gípsicos de origen aluvial (DETENAL, 1981) que pueden tener o no una capa de material aluvial calcáreo de espesor variable. La precipitación anual promedio (estación climática de Vanegas, S.L.P.) de 1980 a 2004 fue de $328 \mathrm{~mm}$ y la temperatura media de $17.6^{\circ} \mathrm{C}$. Durante el tiempo de estudio la precipitación y temperatura medias fueron de $348 \mathrm{~mm}$ y $18.0^{\circ} \mathrm{C}$, respectivamente (Comisión Nacional del Agua, datos no publicados). Las temperaturas mínimas y máximas registradas durante el estudio fueron $-7^{\circ} \mathrm{C}$, (diciembre) y $40^{\circ} \mathrm{C}$ (mayo), respectivamente.

El muestreo se llevó a cabo en la porción oriental del zacatal gipsófilo y los matorrales micrófilos adyacentes. Ahí se establecieron 4 parcelas (Fig. 1): a) Zacatal, que incluyó una parte de la colonia activa de perritos llaneros, con suelo gipsófilo expuesto, desprovisto de arbustos, con predominio de una especie de Chamaesaracha sp. $(1.9 \%$ de cobertura, con base en la determinación de la cobertura de cada planta en 10 cuadros de $1 \times 1 \mathrm{~m}$ ) y una especie de Cruciferae (1.1\%); b) Matorral 1, matorral micrófilo donde las especies arbustivas más abundantes fueron Lycium berlandieri (42\%; la cobertura de matorrales se basó en 10 cuadros de $10 \times 10$ m), Condalia mexicana (13\%), Larrea tridentata (13\%), Opuntia cantabrigensis (11\%), Rhus microphyla (8\%), Opuntia imbricata (6\%) y Yucca sp. (4\%); c) Matorral 2, matorral micrófilo de L. berlandieri (54\%), L. tridentata (28\%), Atriplex canescens (9\%) у O. cantabrigensis (4\%). Ambos matorrales tienen un sustrato base de yeso cubierto por material calcáreo, y d) Arroyo, matorral micrófilo en el lecho de un arroyo intermitente con estrato aluvial profundo, donde la comunidad vegetal está compuesta de Prosopis laevigata (32\%), L. tridentata 


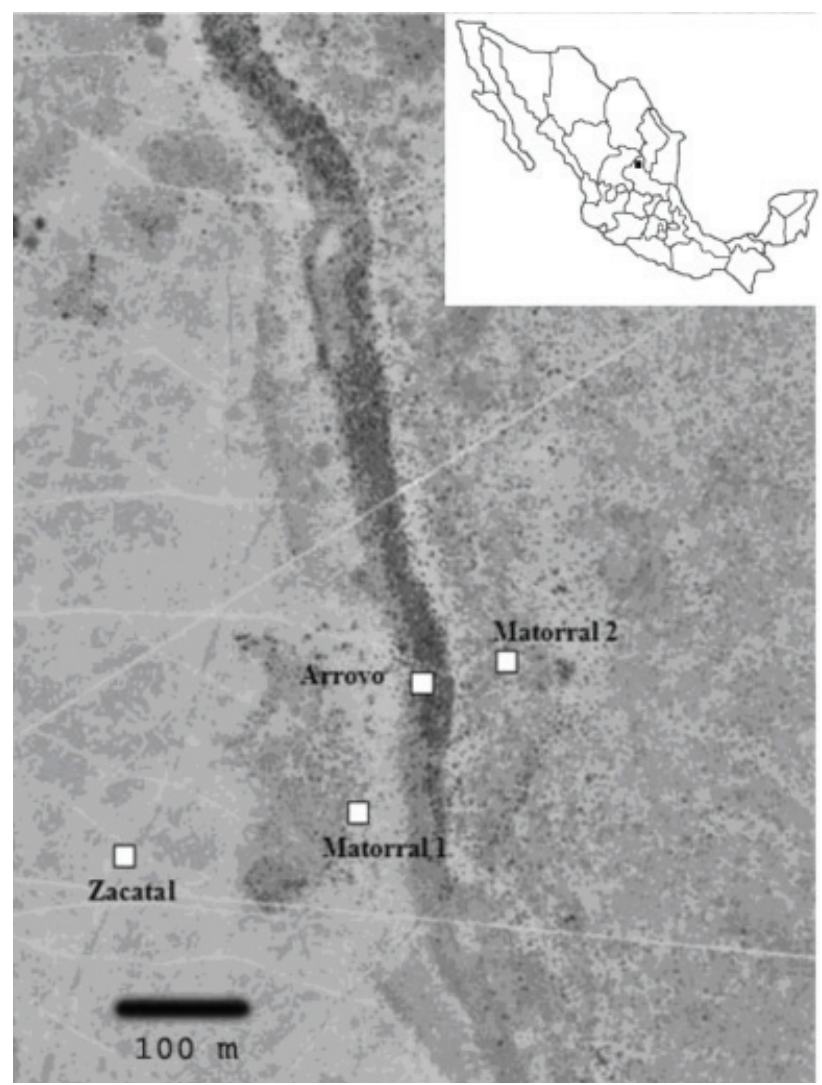

Figura 1. Ecotono entre la extrusión yesífera de El manantial, Vanegas, S.L.P., y matorral micrófilo adyacente, en el que se indican las 4 parcelas de estudio de roedores nocturnos. 20032004.

(28\%), Rhus microphylla (23\%), Condalia mexicana (12\%) y Koeberlinia spinosa $(11 \%)$.

Con base en los promedios de la precipitación y la temperatura ambiental mensual de 24 años, el año se dividió en estación lluviosa caliente (mayo a octubre), estación lluviosa fría (noviembre a enero) y estación seca (febrero a abril). Durante las estaciones lluviosa caliente y lluviosa fría de 2003 y 2004 y la seca de 2004 se estudiaron las comunidades de roedores nocturnos en 7 visitas de muestreo: junio y noviembre de 2003; y abril, junio, julio, septiembre y diciembre de 2004. En cada parcela se colocaron 40 trampas Sherman de $8 \times 9 \times 23 \mathrm{~cm}$, en 4 líneas de 10 trampas, separadas $10 \mathrm{~m}$ entre sí y cebadas con hojuelas de avena, durante 2 noches consecutivas por fecha (2240 noches-trampa). Cada individuo capturado se identifió in situ, se marcó numéricamente por corte de falanges y fue liberado en el sitio de captura.

Dado que las capturas totales de algunas especies de roedores fueron muy bajas, utilizamos el número de individuos capturados en cada periodo como el mejor estimador del tamaño de las poblaciones. Para determinar si había diferencias en la abundancia total de roedores entre los 4 sitios de muestreo a lo largo del estudio, se utilizaron Análisis de Varianza (ANVA) paramétrico con 3 fuentes de variación: especie, muestreo y sitio. Las medias se compararon mediante la prueba de Tukey $(\partial \leq 0.05)$. Se analizaron las diferencias en la abundancia y riqueza específica de cada especie de roedor entre las 4 parcelas a lo largo del estudio. Debido a que estos datos no cumplieron con los requisitos de normalidad y homocedasticidad requeridos para el ANVA paramétrico, se utilizaron ANVAs no paramétricos (pruebas de Friedman; $\partial \leq 0.10$ ), complementadas con pruebas no paramétricas de comparación de medias (Zar, 1999). Los análisis se realizaron con el programa Statistix 7.

Los patrones de comunidades de roedores se estudiaron mediante un análisis de componentes principales. El número de individuos capturados por especie y sitio de muestreo, promediados por época del año, se ordenaron mediante un Análisis de Componentes Principales (ACP).

\section{Resultados}

Durante el estudio capturamos 414 individuos de roedores nocturnos de 12 especies, 6 de heterómidos (3 cuadrúpedas y 3 bípedas) y 6 de múridos (Cuadro 1). Las 6 especies de heterómidos son consideradas granívoras, los múridos Neotoma albigula y Sigmodon hispidus, herbívoros y las especies del género Peromyscus y Reithrodontomys fulvescens, omnívoros. La riqueza total de especies por sitio varió de 2 a 10 (promedio = 7.5; Cuadro 1). Zacatal fue el sitio significativamente menos rico, con sólo 2 especies de roedores, mientras que Matorral 1 fue el más rico, con 10 . No hubo diferencias significativas en la riqueza de roedores entre los sitios de matorral (Arroyo, Matorral 1 y Matorral 2). En Arroyo hubo un incremento en la riqueza de especies en noviembre de 2003 y abril de 2004, que coincidió con un incremento en la cobertura de herbáceas (claramente visible, aunque ésta no se determinó cuantitativamente). Sigmodon hispidus y Chaetodipus nelsoni se capturaron exclusivamente en Arroyo. Sigmodon hispidus se capturó solamente en el mes de noviembre y C. nelsoni se capturó en todos los muestreos pero con mayor abundancia en junio de 2003, un mes después del pico de precipitación (mayo) que produjo un crecimiento importante de la vegetación herbácea.

La mayor abundancia total de roedores se presentó en Arroyo y la menor en Zacatal (Cuadro 1). La abundancia de roedores no fue significativamente diferente entre 1 Arroyo y Matorral 1, como tampoco la de Matorral 1 y 2. La abundancia total en Zacatal fue significativamente 
Cuadro 1. Abundancia total y riqueza de roedores nocturnos, durante 7 periodos en el Manantial, San Luis Potosí, México*

\begin{tabular}{|c|c|c|c|c|}
\hline \multirow[t]{2}{*}{ Especie } & \multicolumn{4}{|c|}{ Sitio } \\
\hline & Arroyo & Matorral 1 & Matorral 2 & Zacatal \\
\hline Perognathus flavus & $0^{\mathrm{b}}$ & $13^{\mathrm{a}}$ & $3^{\mathrm{ab}}$ & $13^{\mathrm{a}}$ \\
\hline Chaetodipus penicillatus & $34^{\mathrm{a}}$ & $42^{\mathrm{a}}$ & $38^{\mathrm{a}}$ & $0^{\mathrm{b}}$ \\
\hline C. nelsoni & $25^{\mathrm{a}}$ & $0^{\mathrm{b}}$ & $0^{\mathrm{b}}$ & $0^{\mathrm{b}}$ \\
\hline Dipodomys merriami & $2^{\mathrm{a}}$ & $33^{\mathrm{a}}$ & $26^{\mathrm{a}}$ & $0^{\mathrm{b}}$ \\
\hline D. ordii & 0 & 6 & 1 & 0 \\
\hline D. nelsoni & $0^{\mathrm{b}}$ & $15^{\mathrm{a}}$ & $5^{\mathrm{ab}}$ & $0^{\mathrm{b}}$ \\
\hline Reithrodontomys fulvescens & 4 & 1 & 0 & 0 \\
\hline Peromyscus truei & $7^{\mathrm{ab}}$ & $8^{\mathrm{ab}}$ & $19^{\mathrm{a}}$ & $0^{\mathrm{b}}$ \\
\hline P. eremicus & $28^{\mathrm{a}}$ & $5^{\mathrm{ab}}$ & $7^{\mathrm{ab}}$ & $0^{\mathrm{b}}$ \\
\hline P. maniculatus & 3 & 2 & 3 & 1 \\
\hline Neotoma albigula & $29^{\mathrm{a}}$ & $3^{\mathrm{bc}}$ & $14^{\mathrm{ab}}$ & $0^{c}$ \\
\hline Sigmodon hispidus & 2 & 0 & 0 & 0 \\
\hline Abundancia total & $1.82 \pm 2.43^{\mathrm{a}}$ & $1.56 \pm 2.19^{\mathrm{ab}}$ & $1.38 \pm 2.12^{\mathrm{b}}$ & $0.17 \pm 0.60^{c}$ \\
\hline Riqueza & $9^{\mathrm{a}}$ & $10^{\mathrm{a}}$ & $9^{\mathrm{a}}$ & $2^{\mathrm{b}}$ \\
\hline
\end{tabular}

*Los valores de cada especie y de riqueza con letra diferente fueron estadísticamente diferentes (Prueba de Friedman, $\partial \leq 0.10)$. Las hileras cuyos valores no incluyen letras, no exhibieron diferencias significativas. Los valores de abundancia total son promedios por periodo y por especie. Aquellos con letra distinta por renglón fueron significativamente diferentes (ANVA de 3 vías, $\partial \leq 0.05$ ).

menor a la de los demás sitios $(\mathrm{p} \leq 0.05)$.

La especie más abundante en todo el estudio fue Chaetodipus penicillatus, con un total de 114 individuos distintos capturados, es decir el $27.5 \%$ del total de capturas. La siguiente especie en importancia fue Dipodomys merriami con 80 capturas $(19.3 \%)$. Con abundancias totales parecidas siguieron $N$. albigula (46 capturas, $11.1 \%$ ), Peromyscus eremicus (40 capturas, 9.7\%) y $P$. truei (37 capturas, 9\%). Las especies con capturas de 20 a 30 fueron Perognathus flavus, C. nelsoni y Dipodomys nelsoni.

Por sitios, en Arroyo, en 3 de las 7 fechas, la especie más abundante fue $P$. eremicus, donde representó entre 27 y $47 \%$ de las capturas totales durante los meses de septiembre a abril. De junio a agosto, esta especie prácticamente no fue capturada en este hábitat y el predominio fue de $N$. albigula, D. merriami y C.penicillatus. En Matorral 1, el promedio de capturas de las 7 fechas fue de 16.6 individuos (DE = 4.3); aquí el predominio de capturas fue de $C$. penicillatus en 5 de las 7 fechas con un 30 a $50 \%$ por arriba de los individuos atrapados. Cuando esta especie no fue la predominante, lo fueron D. merriami o Peromyscus truei. El patrón fue similar en el sitio Matorral 2, donde en 4 de las 7 fechas predominó $C$. penicillatus; hubo un empate entre ésta y D. merriami en 2 casos y en una de las fechas predominó esta última.especie. El pastizal sólo tuvo 2 especies y con poca abundancia (1 a 3 capturas): Perognathus flavus y $P$. maniculatus.

En promedio las capturas fueron más altas en Arroyo, que en Matorral 2 y en éste que en el pastizal. Matorral 1 no fue diferente de Arroyo ni de Matorral 2. Chaetodipus nelsoni sólo se presentó en el sitio Arroyo y sus capturas vinieron a la baja consistentemente desde la primera (7 individuos) hasta la última fecha (una captura). En este mismo hábitat $N$. albigula presentó su mayor abundancia, lo que indica que la cobertura de arbustos mayor la favorece. La ausencia de arbustos y prácticamente de hierbas y pastos en el pastizal yesoso solo permite la presencia limitada de Perognathus flavus y Peromyscus maniculatus.

El análisis de las comunidades de roedores mostró que los primeros 2 ejes del ACP de los sitios explicaron el $48.3 \%$ y el $23.3 \%$ de la varianza, respectivamente (Fig. 
2), para un total acumulado de $71.6 \%$. El componente principal 1 reflejó el efecto del sustrato gipsófilo sobre la estructura general de la vegetación y las comunidades de roedores, separando marcadamente al Zacatal de los 3 sitios de matorral, que se agruparon al otro extremo del eje (Fig. 2). El componente principal 2 sigue laxamente un gradiente de menos a más cobertura de herbáceas.

Al agrupar los datos de las especies de roedores con base en sus abundancias por estación del año y sitio, los primeros 2 ejes del ACP explicaron el 35.7 y $24.5 \%$ de la varianza, respectivamente (Fig. 3). Aparentemente, las especies de roedores se agruparon en función de la cobertura de herbáceas, y el componente principal 1 siguió un gradiente desde una cobertura alta de herbáceas y presencia de arbustivas hasta una cobertura baja de herbáceas y sin arbustos. El eje 2 no se pudo reificar (sensu Marriott, 1974).

\section{Discusión}

Las especies de roedores de El Manantial se distribuyeron de manera discreta en relación con el hábitat, lo que permitió delimitar claramente 2 comunidades: la de zacatal sobre suelo de yeso expuesto y la de los matorrales sobre suelo de yeso cubierto con una capa de material calcáreo. Esto fue notorio en el ordenamiento de los sitios y las especies en el ACP. El ACP que organiza los 4 sitios los separó claramente y reflejó que Zacatal soportaba una comunidad diferente, mucho más pobre, tanto en riqueza como en abundancia, respecto a las 3 comunidades de matorral. Las 2 comunidades de roedores así delimitadas se pueden explicar en función de la estructura y la cobertura vegetal y reflejan su estructura a escala de macrohábitat (Mellink, 1995b; Jorgensen, 2004; Riojas-López, 2006).

La transición entre Zacatal y Matorral 1 y 2 en la composición de especies de roedores fue abrupta ya que en Zacatal sólo se capturaron Perognatus flavus y Peromyscus Maniculatus; de la segunda especie un solo individuo, que quizás se aventuró a salir de la protección de Matorral 2 (que distaba aproximadamente $100 \mathrm{~m}$ de las trampas Sherman). A pesar de ser similares, en el ámbito del estudio los 3 sitios de matorral exhibieron diferencias en sus comunidades de roedores nocturnos. Así, aunque a nivel macro se pueden diferenciar claramente 2 comunidades, entre los matorrales también hay diferencias, aunque no tan marcadas como entre ellos y Zacatal. Entre los matorrales, Matorral 1 y Arroyo se separan claramente, lo que se mantiene a lo largo del año. Por su parte Matorral 2 es más variable y pareció comportase como una comunidad transicional durante el periodo de estudio (Fig. 2).

Arroyo es un sitio con hábitat más cerrado que Matorral

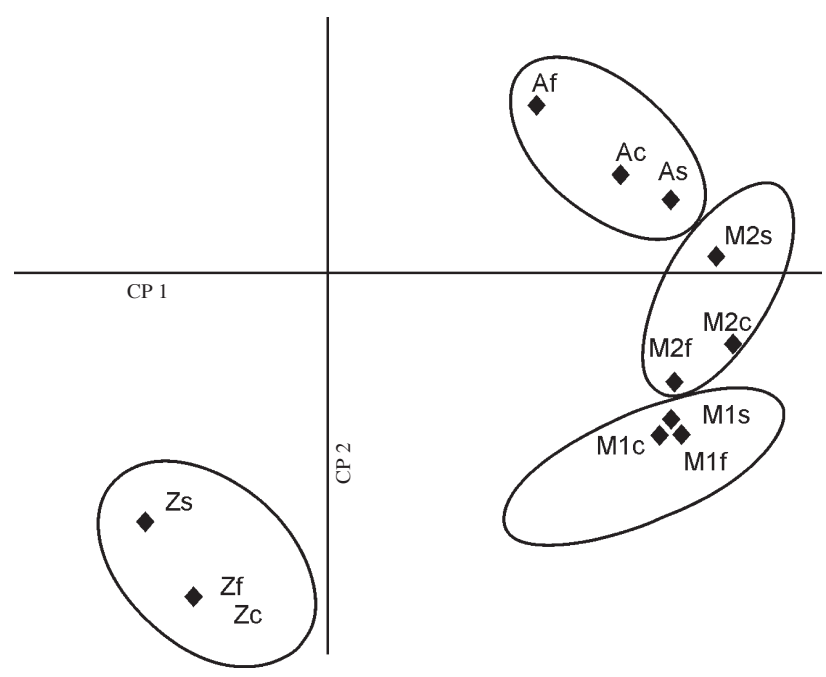

Figura 2. Ordenación de 4 sitios de muestreo y 3 estaciones en la sección oriental de la extrusión yesífera de El Manantial, Vanegas, S.L.P., a lo largo de los 2 primeros ejes de un Análisis de Componentes Principales, basado en la abundancia promedio de 12 especies de roedores nocturnos. $\mathrm{Z}=$ Zacatal, $\mathrm{M} 1=$ Matorral 1, M2 = Matorral 2, A = Arroyo, $\mathrm{c}=$ estación lluviosa caliente (mayo a octubre), $\mathrm{f}=$ estación lluviosa fría (noviembre a enero), y s = estación seca (febrero a abril). 2003-2004.

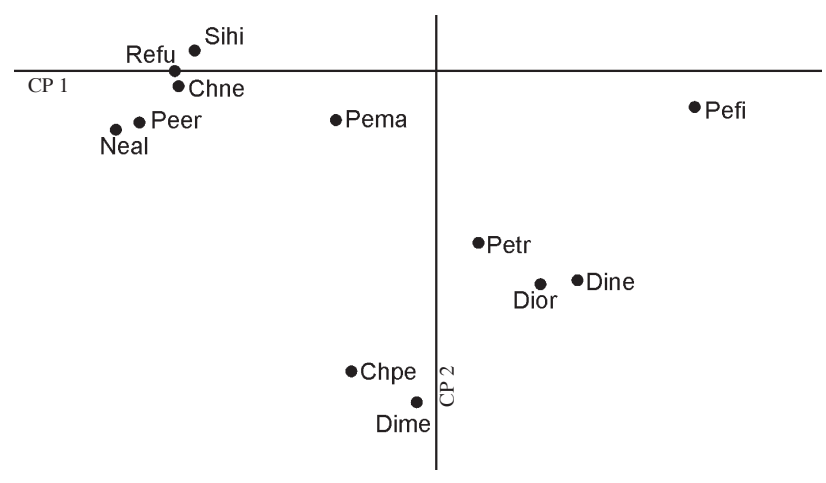

Figura 3. Ordenación de 12 especies de roedores en la sección oriental de la extrusión yesífera de El Manantial, Vanegas, S.L.P., a lo largo de los 2 primeros ejes de un Análisis de Componentes Principales, con base en sus abundancias promedio por sitio y estación. Las abreviaturas de las especies corresponden a las 2 primeras letras del género y las 2 primeras letras de la especie (véase Cuadro 1). 2003-2004.

1 y Matorral 2, donde la vegetación forma manchones bien definidos. El arreglo de los 3 matorrales mediante el ACP puede estar relacionado con efectos de microhábitat sobre las comunidades de roedores (Jorgensen, 2004) en función de la variación en la cobertura de herbáceas, al menos estacionalmente. 
Las afinidades de hábitat se reflejan en el ordenamiento de los roedores con base en sus abundancias en las diferentes fechas de muestreo (Fig. 3). El grupo formado por especies con una alta afinidad por sitios con densidad alta de herbáceas (lado izquierdo de la gráfica), incluyó las que fueron más abundantes en Arroyo: C. nelsoni, S. hispidus y $R$. fulvescens. Las 2 últimas tienden a estar presentes sólo cuando la cobertura de herbáceas es densa (Mellink, 1995b, Riojas-López, 2006), lo que concuerda con su ecología en otros sitios (Spencer y Cameron, 1982; Windberg, 1998).

El hecho que $P$. flavus haya sido la única especie presente de manera regular en el zacatal gipsófilo contrasta con un estudio previo (Mellink y Madrigal, 1993), donde se capturó a Dipodomys ordii, Onychomys arenarius, $D$. nelsoni y Peromyscus sp. en secciones abandonadas y activas de la colonia de perritos llaneros en este mismo sitio. También contrasta con las comunidades encontradas en otras colonias de perritos llaneros (C. ludovicianus), en los pastizales de Janos, Chihuahua., donde se encontraron 7 especies de ratones (Pacheco et al., 2000), lo cual se puede explicar por diferencias en la cobertura vegetal, y que coincide con otros estudios, en los que la disminución o eliminación de la cobertura de los pastos causa empobrecimiento de la comunidad de roedores (Stapp, 2007). La cobertura de herbáceas registrada en dicho estudio (1988) fue de 40\%, mientras que en 2003 registramos del 4.5-9.3\% de cobertura de herbáceas en el área. En 1988 Muhlenbergia purpussi (indicada como $M$. repens) fue la especie con mayor cobertura en la colonia (24-36\% de cobertura), pero en 2003 esta especie sólo se encontraba formando manchones aislados dentro de Zacatal y estuvo ausente del área muestreada.

Los cambios en la precipitación en un lapso de 24 años quizás puedan explicar las diferencias en la vegetación de Zacatal. En el periodo de 1986-1988 tuvieron una precipitación promedio de $507.33 \mathrm{~mm}$, lo cual pudo haber influido en la cobertura reportada en el estudio mencionado de 1988. La precipitación promedio de 1989 al 2004 fue de 291.76 con años atípicos, como el de 1992 con $661.8 \mathrm{~mm}$ y el de 1999 con $72 \mathrm{~mm}$. En los 3 años previos al estudio la precipitación promedio fue de $294 \mathrm{~mm}$, que pudo tener como resultado las coberturas vegetales registradas en este trabajo.

Otro factor aparente que puede estar relacionado con estos cambios es el pastoreo intenso por cabras y borregos en años recientes. Durante el tiempo de estudio se observaron hatos de hasta 500 animales en Zacatal. Los cambios en la vegetación entre 1988 (Mellink y Madrigal, 1993) y 2003 (este estudio) fueron de cobertura, pero no de composición de especies de plantas, lo cual contrasta con observaciones en otros sistemas no gipsófilos (Fleischner, 1994), donde los cambios de la vegetación debidos al pastoreo son tanto cualitativos (especies) como cuantitativos (abundancia). Sin embargo, contrastando con un estudio de las comunidades vegetales de suelos gipsófilos próximas al sitio de estudio, que documentó que estas comunidades vegetales se mantienen estables aún en presencia de pastoreo (Meyer y García-Moya, 1989), Zacatal no cambió en composición de especies de plantas, pero si en la cobertura. El pastoreo puede afectar las poblaciones de roedores de diferente forma, dependiendo de sus efectos específicos sobre la comunidad vegetal (Bock et al., 1984; Jones y Longland, 1999; Fleischner, 1994; Mellink y Valenzuela, 1995). El pastizal gipsófilo de El Manantial parece haber sido afectado a grado tal que ocasionó un empobrecimiento fuerte de la comunidad de roedores. Si la intensidad de este disturbio se aminora se esperaría que se volviera a las comunidades encontradas en 1988 (Mellink y Madrigal 1993).

En conclusión, 1), las comunidades de roedores nocturnos de los paisajes yesosos de San Luis Potosí muestran una relación estrecha con la vegetación, y cambiaron de manera notable entre Zacatal y los 3 sitios de matorral adyacentes; 2), la composición y la abundancia de roedores nocturnos presentó variación más vinculada al mosaico de la vegetación que a la época del año; 3), las comunidades de ratones nocturnos son muy parecidas entre los matorrales, pero mantienen su identidad individual a lo largo del año y 4), una baja precipitación en los años previos al estudio y/o el pastoreo intenso y prolongado en este sistema condujo a una reducción significativa de la cobertura de pastos y herbáceas, lo que causó un cambio en la composición, abundancia y disminución de la riqueza en las comunidades de roedores nocturnos.

\section{Agradecimientos}

Al Dr. J. Rogelio Aguirre, Director del Instituto de Investigaciones Desérticas (IIZD, Universidad Autónoma de San Luis Potosí) y al Centro de Investigación Científica y de Educación Superior de Ensenada (CICESE), por su apoyo logístico a lo largo del estudio. A Andrés Luévano Luna y Pedro Luévano Santos, por su compañía y ayuda en el trabajo de campo. Un revisor anónimo ayudó a mejorar este artículo. El Consejo Nacional de Ciencia y Tecnología (CONACYT) apoyó al primer autor con una beca. La mayor parte del financiamiento de este estudio provino de la familia Luévano Luna. 


\section{Literatura citada}

Archer, S. 1995. Herbivore mediation of grass-woody plant interactions. Tropical Grasslands 29:218-235.

Bock, C. E., J. H. Bock, W. R. Kenney y V. M. Hawthorne. 1984. Responses of birds, rodents and vegetation to livestock exclosure in a semidesert grassland. Journal of Range Management 37:239-244.

Brown, J. H. y E. Heske. 1990. Control of a desert-grassland transition by a keystone rodent guild. Science 250:17051707.

Ceballos, G., E. Mellink y L. Hanebury. 1993. Distribution and conservation status of prairie dogs Cynomys mexicanus and Cynomys ludovicianus in Mexico. Biological Conservation 63:105-112.

DETENAL. 1981. Atlas nacional del medio físico de los Estados Unidos Mexicanos. Secretaría de Programación y Presupuesto, México, D.F.

Fleischner, T. L. 1994. Ecological costs of livestock grazing in Western North America. Conservation Biology 8:629-644.

Guo, Q., D. B. Thompson, J. Valone y J. H. Brown. 1995. The effects of vertebrate granivores and folivores on plant community structure in the Chihuahuan Desert. Oikos 73:251-259.

Kay, F. R. y W. G. Whitford. 1978. Burrow environment of the banner-tailed kangaroo rat, Dipodomys spectabilis, in south-central New México. American Midland Naturalist 99:270-279.

Jones, A. L. y W. Longland. 1999. Effects of cattle grazing on salt rodent communities. American Midland Naturalist 141:1-11.

Jorgensen, E. E. 2004. Small mammal use of microhabitats reviewed. Journal of Mammalogy 85:531-539.

Marriott, F. H. C. 1974. The interpretation of multiple observations. Academic, New York. 117 p.

Mellink, E. 1995a. Fauna silvestre y ganadería extensiva: ¿antagonistas? Memorias del 1er reencuentro de Zootecnistas. Universidad Autónoma Chapingo, Chapingo, Estado de México. p. 33-47

Mellink, E. 1995b. Uso del hábitat, Dinámica poblacional y estacionalidad reproductiva de roedores en el Altiplano Potosino, México. Revista Mexicana de Mastozoología1: 1-8.

Mellink, E. y H. Madrigal. 1993. Ecology of Mexican praire dogs, Cynomys mexicanus, in El Manantial, Northeastern Mexico. Journal of Mammalogy 74:631-635.

Mellink, E. y S. Valenzuela. 1992. Comunidades aviares y su modificación por el pastoreo en agostaderos del municipio de Salinas, S. L. P. Agrociencia, Serie Recursos Naturales Renovables 2:87-94.

Mellink, E. y S. Valenzuela. 1995. Efecto de la condición de agostaderos sobre los roedores y lagomorfos en el Altiplano Potosino, San Luis Potosí, México. Acta Zoologica Mexicana 64:35-44.

Meyer, S. E. y E. García- Moya. 1989. Plant community patterns and soil moisture regime in gypsum grasslands of north central México. Journal of Arid Environments 16:147-155.

Meyer, S. E., E. García- Moya y L. C. Lagunes-Espinoza. 1992. Topographic and soil moisture regime in gypsum grassland of north central México. Journal of Vegetation Science $3: 429-438$.

Pacheco, J. G. Ceballos y R. List. 1999-2000. Los mamíferos de la región de Janos, Casas Grandes, Chihuahua, México. Revista Mexicana de Mastozoología 4:69-83.

Price, M. 1978. The role of microhabitat in structuring desert rodent communities. Ecology 59:910-921.

Price, M. y N. Waser. 1984. On the relative abundance of species post fire changes in a coastal sage shrub rodent community. Ecology 65:1161-1169.

Riojas-López, M. E. 2006. Rodent communities in two natural and one cultivated "nopaleras" (Opuntia spp.) in northeastern Jalisco, Mexico. Journal of Arid Environments 67:428-435.

Rosenzweig, M. L y J. Winakur. 1969. Population ecology of desert rodent communities: habitat and environment complexity. Ecology 50:558-572.

Rzedowski, J. 1955. Notas sobre la flora y la vegetación del estado de San Luis Potosí. 2. Estudio de diferencias florísticas y ecológicas condicionadas por ciertos tipos de sustrato geológico. Ciencia (México) 15:141-158.

Spencer, S. R. y G. N. Cameron. 1982. Reithrodontomys fulvescens. Mammalian Species 174:1-7.

Stapp, P. 2007. Rodent communities in active and inactive colonies of black-tailed prairie dogs in shortgrass steppe. Journal of Mammalogy 88:241-249.

Windberg, L. A. 1998. Population trends and habitat associations of rodents in Southern Texas. American Midland Naturalist 140:153-160.

Yeaton, R. I. y J. L. Flores-Flores. 2006. Patterns of occurrence and abundance in colony complexes of the Mexican prairie $\operatorname{dog}$ (Cynomys mexicanus) in productive and unproductive grasslands. Acta Zoologica Mexicana n.s. 22:107-130.

Zar, J. H. 1999. Biostatistical analysis, cuarta edición. Prentice Hall, Englewood Cliffs, New Jersey. 663 p. + apéndices. 